\title{
Chemoimmunotherapy resistant follicular lymphoma - A single institutional study
}

\author{
Sampath Kumar-Kandala Jeevan ${ }^{1}$, Roshni Paul-Tara ${ }^{1}$, Shantiveer Uppin ${ }^{1}$, Megha Uppin ${ }^{1}$, \\ Kavitha Bali ${ }^{2}$, Sundaram-Challa ${ }^{1}$, Raghunadha Rao $^{3}$ \\ ${ }^{1}$ Department of Pathology, Nizam`s Institute of Medical Sciences, Hyderabad, India \\ ${ }^{2}$ Department of Pathology, Osmania Medical College, Hyderabad, India \\ ${ }^{3}$ Department of Medical Oncology, Nizam`s Institute of Medical Sciences, Hyderabad, India
}

\section{Email address:}

kmcsampath@gmail.com (Sampath K. K. J.)

\section{To cite this article:}

Sampath Kumar-Kandala Jeevan, Roshni Paul-Tara, Shantiveer Uppin, Megha Uppin, Kavitha Bali, Sundaram-Challa, Raghunadha Rao. Chemoimmunotherapy Resistant Follicular Lymphoma - A Single Institutional Study. Cancer Research Journal.

Vol. 2, No. 5, 2014, pp. 93-97. doi: 10.11648/j.crj.20140205.13

\begin{abstract}
Follicular lymphoma (FL) is the most common indolent form of non Hodgkin lymphoma and characterized by initial response to treatment with inevitable relapse. Rituximab, is an anti CD20 monoclonal antibody, plays an important role in combination chemoimmunotherapy in treatment of symptomatic follicular lymphoma. Though rituximab is helpful in reducing the tumor burden in patients of follicular lymphoma, a significant proportion of patients were found to have resistance to rituximab and eventually progressed to high grade lymphoma. In this study we evaluated chemoimmunotherapy (CIR) resistant patients to know the incidence, predictive factors associated with CIR resistance and prognosis. This is a retrospective study, included 49 patients of follicular lymphoma, of these $12(24.5 \%)$ demonstrated CIR resistance. On univariate analysis, high LDH values, advanced stage and high FLIPI score may predict the resistance of CIR therapy in initial stage of therapy. Overall, 7 patients (58.3\%) progressed to high grade lymphoma (biopsy proven) and $2(16.6 \%)$ died of disease progression in CIR resistant patients. Progressive free survival significantly reduced in CIR resistant patients (26 months) than CIR responders.
\end{abstract}

Keywords: Chemoimmunotherapy Resistance, Follicular Lymphoma, FLIPI Score, Lactate Dehydrogenase, Advance Stage, Progression

\section{Introduction}

Follicular lymphoma (FL) is an indolent non hodgkin lymphoma characterized by an initial response to treatment but inevitable relapse. In the last decade, immunotherapy with anti CD20 monoclonal antibody, Rituximab, modified the therapeutic approach of B-cell non-hodgkin lymphoma(1). Conventional chemotherapy does not alone cure the follicular lymphoma but combining the chemotherapy with monoclonal antibody therapy has better results to the same chemotherapy alone, so concomitant chemoimmunotherapy has become first line treatment for follicular lymphoma(2). However, a subset of patients will show disease progression during initial Chemoimmunotherapy or maintenance phase of rituximab based therapy. The true incidence of chemoimmunotherapy resistant to rituximab is unknown, estimates range from
$14 \%$ in patients receiving rituximab as a part of primary therapy to $28 \%$ for those receiving chemoimmunotherapy and maintenance rituximab as second line treatment $(3,4,5)$. Disease progression during treatment may result from resistance to chemotherapy, rituximab or to combined therapy. Mechanisms of resistance to rituximab have not been fully elucidated but may include the over-expression of complement inhibitory molecules CD55 and CD59 [6-7)], under-expression of CD20 (8-10) and decreased affinity of rituximab to the $\mathrm{Fc}$ receptor resulting in reduced antibody-dependent cell-mediated cytotoxicity $(11,12)$.

Transformation of follicular lymphoma to a more aggressive histology occurs at an annual rate of 3\% from date of diagnosis, with a 10 -year risk of approximately $30 \%$ in the pre-rituximab era (13). Transformation confers a poor prognosis with a median post-transformation survival of 1 to 2 years (13) and 5- year survival of 25\% (13-16). Main aim 
of our study is to determine the incidence, predictive factors association with histological transformation and outcome of CIR resistance in rituximab therapy.

\section{Material and Methods}

Patients were retrospectively identified using clinical database from single center. Patients with a diagnosed of FL grade1, 2 and 3 a according to the WHO classification were included in this study. Exclusion criteria included composite or discordance lymphoma and FL grade $3 \mathrm{~b}$. Baseline characteristics were recorded and included histology, advanced stage disease (stage III or IV) FLIPI risk score.

\subsection{Chemoimmunotherapy Resistance Definition}

CIR resistance was defined as either resistance to initial chemoimmunotherapy or to maintenance rituximab within 6 months of treatment completion.

\subsection{Transformation Definition}

Determination of transformation was based on a biopsy demonstrating FL grade 3b, diffuse large B-cell lymphoma (DLBCL), Burkitt lymphoma (BL) or B-cell lymphoma unclassifiable with features intermediate between DLBCL and BL as per WHO 2008 classification (17).

\subsection{Outcome Definition}

PFS was measured from initiation of rituximab-based chemoimmunotherapy to radiological or clinical progression as determined by the treating clinician/oncologist. OS was measured from the initiation of rituximab chemoimmunotherapy until last follow-up or death. PFS was calculated using the Kaplan-Meier method. Chi-square test was used for univariate calculation of clinically relevant predictors for early progression. All statistical calculations were made using Graph Pad Prism, version 5.00 software.

\section{Results}

This retrospective study comprises 68 cases of follicular lymphomas included over a period of four years (2007-2011) in our institute. Nineteen patients were excluded from this study for the following reasons: fifteen patients were lost to follow-up and four patients were treated as diffuse large B-cell lymphoma. Therefore, 49 patients were identified as receiving rituximab based chemoimmunotherapy. Of these, twelve patients $(24.5 \%)$ were resistant to immunochemotherapy. Of these CIR resistance patients, seven were progressed during initial chemoimmunotherapy, three during maintenance therapy and two within 6 months after maintenance completion. From initiation of rituximab containing chemoimmunotherapy, median follow-up was 36 months (range from 6 months to 60months). Baseline characteristics of $\mathrm{CI}$ responder and CIR resistant group described in Table-1.

\subsection{Clinical and Characteristics of Chemoimmunotherapy Resistance Patients}

Of 49 patients, twelve $(24.5 \%)$ patients developed resistant to combination of rituximab and chemotherapy. There was a male predominance with $\mathrm{M}$ : F ratio of 1.4: 1 . All CIR resistant patients $(24.5 \%)$ were biopsied at the time of disease progression and all these patients were demonstrating evidence of disease progression. Of 12 patients, 5 were confirmed to have diffuse large B-cell lymphoma and 2 were B-cell lymphoma unclassifiable with features of DLBCL and Burkitt lymphoma. Outcome and follow-up of CIR resistant with disease progression are shown in Table-2. In univariate analysis, rituximab based chemoimmunotherapy resistance group had statistically significance in association with high lactate dehydrogenase enzyme value $(p=0.0005$, figure- $1 \mathrm{~A})$, advanced stage $(p=<0.0001$, figure-1B) and high FLIPI score $>3(p=0.0005$, figure-1C). Factors which were not statistically significant include, gender $(\mathrm{p}=1.0)$, histological grade $(\mathrm{p}=0.4589)$. Of 12 patients who resistance to therapy, eight achieved partial remission with $\mathrm{R}-\mathrm{CHOP}$ treatment and remaining three were not responded to the treatment. During follow-up, 2 deaths occurred in CIR resistant group.

Table 1. Baseline characteristics of CIR responder and CIR resistant group

\begin{tabular}{lll}
\hline Characteristics & $\begin{array}{l}\text { CIR responder } \\
(\mathbf{n}=\mathbf{3 7})\end{array}$ & $\begin{array}{l}\text { CIR resistance } \\
(\mathbf{n}=\mathbf{1 2})\end{array}$ \\
\hline $\begin{array}{l}\text { Age } \\
\text { Gender (female) }\end{array}$ & 56 & 58.3 \\
Histological grade & $43.2 \%$ & $41.6 \%$ \\
Grade 1 & $22(59.4 \%)$ & $6(50 \%)$ \\
Grade 2 & $13(35.1 \%)$ & $4(33.3 \%)$ \\
Grade 3a & $2(5.4 \%)$ & $2(16.6 \%)$ \\
Bone marrow involvement & $20(54 \%)$ & $9(75 \%)$ \\
FLIP score & $24(64.8)$ & $2(16.6 \%)$ \\
Low (0,1,2) & $10(27 \%)$ & $3(25 \%)$ \\
Intermediate (3) & $3(8.1 \%)$ & $7(58.8 \%)$ \\
High (4 or 5) & $26(70.2 \%)$ & $12(100 \%)$ \\
Stage 3 or 4 & No & $7(58.3 \%)$ \\
High grade lymphoma & & \\
transformation & & \\
\hline
\end{tabular}

Abbreviations: CIR responder - chemoimmunotherapy or maintenance rituximab responder, CIR resistance- chemoimmunotherapy or maintenance rituximab resistance.

\section{Discussion}

Rituximab is most widely used anti-CD20 monoclonal antibody, and has dramatically improved outcomes for patients with follicular lymphoma (1). This monoclonal antibody (rituximab), when combined with chemotherapy it will improves the both response rate and the progression free survival (rituximab for the treatment) in relapse or resistant FL. Although rituximab is useful in untreated and relapse follicular lymphoma, however few patients develop resistance to rituximab along with histological transformation $(13,18)$ into high grade lymphoma, which remains clinical challenge for most of the clinicians. In this 
study were trying to discuss the factors which are associated with resistance and transformation of follicular lymphoma and also prognosis of chemoimmunotherapy resistant patients. Our primary aim was not to distinguish between rituximab with combination therapies and rituximab therapy alone.

This is a retrospective study, comprising of 49 patients of symptomatic follicular lymphoma. Of these, $24.5 \%$ of patients were showing resistant to rituximab combination therapy. Incidence of our study is slight higher than recently published study (2014). There were studies showed that measures of tumor burden includes, FLIPI score and stage of the disease correlate with disease progression $(13,14,15)$. High FLIPI score, high serum LDH and advanced stage were predictive of CIR resistance in univariate analysis in this study, which is similar to Mozessohn et al study and other studies (18). But gender and histological grade were not reached the statistical significance in univariate analysis and this is probably due to small study group.

Not surprisingly, patients with chemoimmunotherapy resistance were found to have with transformation and reduced progressive free and overall survival. Al-Tourah et al (13) reported that rituximab with combination chemotherapy for follicular lymphoma the five year transformation rate was less than 10\%. Mozessohn et al (18) reported the rate of transformation was $36.3 \%$ in those receiving chemoimmunotherapy during or after 6 months of maintenance of rituximab. But in our study, we found much higher rate of transformation of $58.3 \%$ than previously described studies $(13,18)$.

Table 2. Characteristic features and prognosis of CIR resistant patients

\begin{tabular}{|c|c|c|c|c|c|c|c|c|}
\hline Patient & $\begin{array}{l}\text { Age at } \\
\text { diagnosis }\end{array}$ & $\begin{array}{l}\text { Biopsy proven } \\
\text { transformation }\end{array}$ & $\begin{array}{l}\text { Timing of } \\
\text { resistance }\end{array}$ & $\begin{array}{l}\text { Pathology at } \\
\text { transformation }\end{array}$ & $\begin{array}{l}\text { BM } \\
\text { involvement }\end{array}$ & $\begin{array}{l}\text { Treatment after } \\
\text { post } \\
\text { transformation }\end{array}$ & $\begin{array}{l}\text { Response to } \\
\text { treatment post } \\
\text { transformation }\end{array}$ & Death \\
\hline 1 & 58 & Yes & $\begin{array}{l}\text { During initial } \\
\text { therapy }\end{array}$ & N/A & Yes & R-CHOP X4 & PD & Yes \\
\hline 2 & 61 & Yes & After MR & DLBCL & Yes & R-CHOP X6 & PR & No \\
\hline 3 & 56 & Yes & During MR & N/A & No & R-CHOP X6 & PR & No \\
\hline 4 & 62 & Yes & $\begin{array}{l}\text { During } \\
\text { Initial } \\
\text { therapy }\end{array}$ & $\begin{array}{l}\text { Large B-cell } \\
\text { lymphoma }\end{array}$ & Yes & R-CHOP X6 & PR & No \\
\hline 5 & 60 & Yes & $\begin{array}{l}\text { During initial } \\
\text { therapy }\end{array}$ & DLBCL & Yes & R-CHOP X6 & PR & No \\
\hline 6 & 45 & Yes & $\begin{array}{l}\text { During initial } \\
\text { therapy }\end{array}$ & DLBCL & Yes & R-CHOP X6 & PR & No \\
\hline 7 & 70 & Yes & During MR & $\begin{array}{l}\text { Large B-cell } \\
\text { lymphoma }\end{array}$ & Yes & R-CHOP X6 & PR & No \\
\hline 8 & 70 & Yes & During MR & N/A & Yes & R-CHOP X6 & PR & No \\
\hline 9 & 73 & Yes & $\begin{array}{l}\text { During initial } \\
\text { therapy }\end{array}$ & DLBCL & Yes & R-CHOP X2 & PD & Yes \\
\hline 10 & 58 & Yes & $\begin{array}{l}\text { During initial } \\
\text { therapy }\end{array}$ & DLBCL & Yes & R-CHOP X6 & PR & No \\
\hline 11 & 38 & Yes & $\begin{array}{l}\text { During initial } \\
\text { therapy }\end{array}$ & N/A & No & R-CHOP X6 & PD & No \\
\hline 12 & 55 & Yes & After MR & N/A & No & R-CHOP X6 & PD & No \\
\hline
\end{tabular}

Abbreviations: DLBCL - Diffuse large B-cell lymphoma, MR - maintenance rituximab, PD- progressive disease, PR-partial remission, R-CHOP-rituximab, cyclophosphamide, vincristine, doxorubicin, prednisone
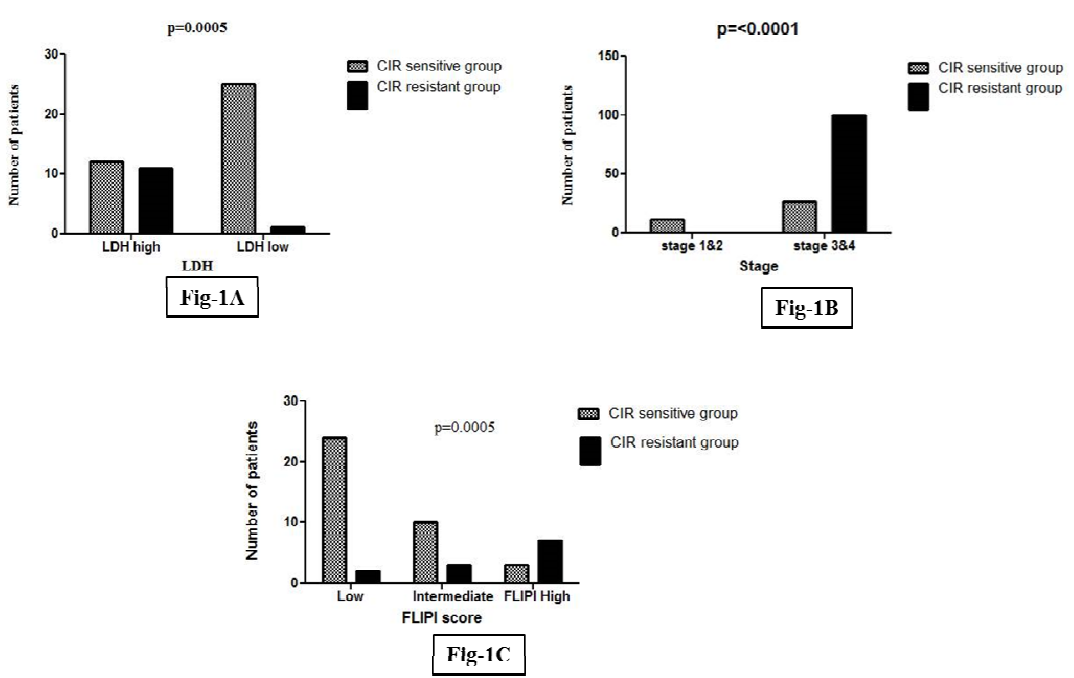

Fig 1. Comparison of LDH levels (Fig-1A), Stage (Fig-1B) and FLIPI Score(Fig-1C) of the disease among CIR responder and resistant group 
Studies showed that R-CHOP regimen with long follow-up found to have improved progressive free survival and decrease the tumor burden relapsed or resistant follicular lymphoma. Marinus et al (5), they randomly assigned 465 patients to induction with either six cycles of CHOP or $\mathrm{R}-\mathrm{CHOP}$ and those in complete remission or partial remission after induction were randomly assigned to maintenance treatment with rituximab. In their study, they showed that significantly increased progressive free survival in R-CHOP group (3.7 years) than observation group (1.8 years) and also confirmed that superior PFS with rituximab in resistant or relapse FL. However studies showed that prolonged maintenance therapy with rituximab resulting in relapse or resistance in follicular lymphoma and the possible mechanisms for resistance to rituximab is due to mutations in CD20 gene (20), over-expression of complement inhibitory molecules CD55 and CD59 [6-7)], under-expression of CD20 (8-10). Marinus et al reported that progression and relapse rate of $35 \%$ to $40 \%$ occurring during or within 6 months of after maintenance of rituximab treatment.

In our study, chemoimmunotherapy resistant patients had taken R-CHOP regimen as a first line of treatment. Of these patients, eight patients $(66.6 \%)$ showed partial response to treatment and remaining patients $(33.3 \%)$ were not responded to treatment and two were died because of disease progression. Compared to Chemoimmunotherapy responder group, in CIR resistant group had significantly reduced progressive free survival (26 months vs. not reached, $\mathrm{p}=<0.0001$, figure-4), which is higher than Mozessohn et al study (18) and overall survival did not reach statistical significance $(\mathrm{p}=0.0561)$, probably due to small study population group.

\section{Conclusion}

Despite of rituximab based chemoimmunotherapy, a significant proportion of patients are developing resistant to treatment and disease progression. In this study, high LDH values, advanced stage and high FLIPI score may predict the risk of resistance to chemoimmunotherapy at the time initial treatment. Progressive free survival was significantly reduced in CIR resistant patients than CI respond patients. So this is time to discover newer regimen to overcome the resistance to rituximab based therapy and also to decrease the rate of disease progression of follicular lymphoma.

\section{References}

[1] Buske C, Gisselbrecht C, Gribben J, et al. Refining the treatment of follicular lymphoma: Leukemia and Lymphoma, 2008; 49:18-26.

[2] Hiddemann W, Kneba M, Dreyling M, et al. - Frontline therapy with rituximab added to the combination of cyclophosphamide, doxorubicin, vincristine and prednisone (CHOP) significantly improves the outcome for patients with advanced-stage follicular lymphomas compared with therapy with $\mathrm{CHOP}$ alone: results of a prospective randomized study of the German Low-Grade Lymphoma Study Group. Blood, $2005 ; 106: 3725-3732$

[3] Salles G, Seymour JF, Offner F et al. Rituximab maintenance for 2 years in patients with high tumour burden follicular lymphoma responding to rituximab plus chemotherapy (PRIMA): a phase 3, randomized controlled trial. Lancet 2011;377:42-51.

[4] van Oers MH, Klasa R, Marcus RE et al. Rituximab maintenance improves clinical outcome of relapsed/resistant follicular non-Hodgkin lymphoma in patients both with and without rituximab during induction: results of a prospective randomized phase 3 intergroup trial. Blood 2006;108:3295-3301.

[5] Marinus H.J, Van Oers MH, Van Glabbeke $\mathrm{M}$ et al. Rituximab maintenance treatment of relapsed/resistant follicular non-Hodgkin's lymphoma: long-term outcome of the EORTC 20981 phase III randomized intergroup study. J Clin Oncol 2010;28:2853-2858.

[6] Golay J, Zaffaroni L, Vaccari T et al. Biologic response of B lymphoma cells to anti-CD20 monoclonal antibody rituximab in vitro: $\mathrm{CD} 55$ and $\mathrm{CD} 59$ regulate complement-mediated cell lysis. Blood 2000;95:3900-3908.

[7] Macor P, Tripodo C, Zorzet $\mathrm{S}$ et al. In vivo targeting of human neutralizing antibodies against CD55 and CD59 to lymphoma cells increases the antitumor activity of rituximab. Cancer Res 2007;67:10556-10563.

[8] Treon SP, Mitsiades C, Mitsiades N et al. Tumor cell expression of CD59 is associated with resistance to CD20 serotherapy in patients with B-cell malignancies. J Immunother 2001;24:263-271.

[9] Czuczman MS, Olejniczak S, Gowda A et al. Acquirement of rituximab resistance in lymphoma cell lines is associated with both global CD20 gene and protein down-regulation regulated at the pretranscriptional and posttranscriptional levels. Clin Cancer Res 2008;14:1561-1570.

[10] Itala M, Geisler CH, Kimby E et al. Standard-dose anti-CD20 antibody rituximab has efficacy in chronic lymphocytic leukaemia: results from a Nordic multicentre study. Eur J Haematol 2002; 69:129-134

[11] Manches O, Lui G, Chaperot L et al. In vitro mechanisms of action of rituximab on primary non-Hodgkin lymphomas. Blood 2003; 101:949-954.

[12] Cartron G, Dacheux L, Salles G et al. Therapeutic activity of humanized anti-CD20 monoclonal antibody and polymorphism in IgG Fc receptor FcgammaRIIIa gene. Blood 2002; 99:754-758.

[13] Czuczman MS, Gregory SA. The future of CD20 monoclonal antibody therapy in B-cell malignancies. Leuk Lymphoma 2010; 51:983-994.

[14] 13. Al-Tourah AJ, Gill KK, Chhanabhai $\mathrm{M}$ et al. Population-based analysis of incidence and outcome of transformed non-Hodgkin's lymphoma.J Clin Oncol 2008;26:5165-5169.

[15] Montoto S, Davies AJ, Matthews J et al. Risk and clinical implications of transformation of follicular lymphoma to diffuse large B-cell lymphoma. J Clin Oncol 2007; 25:2426-2433. 
97 Sampath Kumar-Kandala Jeevan et al: Chemoimmunotherapy Resistant Follicular Lymphoma - A Single Institutional Study

[16] Bastion Y, Sebban C, Berger F et al. Incidence, predictive factors, and outcome of lymphoma transformation in follicular lymphoma patients. J Clin Oncol 1997; $15: 1587-1594$

[17] Solal-Celigny P, Roy $\mathrm{P}$, Colombat $\mathrm{P}$ et al. Follicular lymphoma international prognostic index. Blood 2004; 104:1258-1265.

[18] Swerdlow S, Campo E, Harris N et al. WHO Classification of Tumours of Haematopoietic and Lymphoid Tissues. Lyon, France: IARC Press 2008.
[19] L. Mozessohn, M.C. Cheung, M. Crump et al. Chemoimmunotherapy resistant follicular lymphoma: predictors of resistance, association with transformation and prognosis. Leukemia \& lymphoma 2014 Jan: (doi:10.3109/10428194.2014.885513).

[20] Hatake K, Terui. Rituximab resistance in B-cell lymphoma and its elimination. Gan to kagaku ryoho cancer \& chemotherapy; 2009 Apr; 36 (4):548-51. 